\title{
Multicentric Inverted Papilloma of Sinonasal Region and the Temporal Bone
}

\author{
${ }^{1} \mathrm{MC}$ Trupthi, ${ }^{2}$ Mary John, ${ }^{3}$ Rajavel Subbaraj, ${ }^{4}$ Ajoy M Varghese
}

\begin{abstract}
Inverted papilloma is a benign but a locally aggressive sinonasal tumor. Inverted papilloma of the temporal bone, though rare, has been reported to occur either primarily or secondarily. Here we present a case of recurrent sinonasal inverted papilloma with synchronous lesion of the middle ear, having no connection through the eustachian tube.
\end{abstract}

Keywords: Inverted papilloma, Middle ear, Recurrent, Synchronous.

How to cite this article: Trupthi MC, John M, Subbaraj R, Varghese AM. Multicentric Inverted Papilloma of Sinonasal Region and the Temporal Bone. Int J Otorhinolaryngol Clin 2018;10(1):35-38.

Source of support: Nil

Conflict of interest: None

\section{INTRODUCTION}

Inverted papilloma is a benign but locally aggressive tumor of the sinonasal cavity. Inverted papilloma represents only 0.5 to $4 \%$ of all sinonasal neoplasms. ${ }^{1}$ Only 33 cases of inverted papilloma of the temporal bone have been reported in literature, out of which 18 had tumor limited to the temporal bone and 14 had extension from the sinonasal cavity with one study not mentioning the sinonasal involvement clearly. ${ }^{2}$ To our knowledge, there is no literature reporting a case of inverted papilloma involving the sinonasal cavity and the temporal bone with no communication through the eustachian tube.

Kramer and Som ${ }^{3}$ classified inverted papilloma as true nasal papilloma and differentiated it from inflammatory nasal polyps. The tendency of inverted papilloma for local aggressiveness was described by Ringertz. ${ }^{4}$ Inverted papilloma is clinically characterized by its local aggressiveness, high recurrence rate, associated malignancy, and tendency to multicentricity which necessitates radical surgical removal of these tumors.,

\footnotetext{
${ }^{1,3}$ Postgraduate Student, ${ }^{2,4}$ Professor

${ }^{1-4}$ Department of ENT, Christian Medical College, Vellore, Tamil Nadu, India
}

Corresponding Author: MC Trupthi, Postgraduate Student Department of ENT, Christian Medical College, Vellore, Tamil Nadu, India, Phone: +919159593719, e-mail: trupthi.mc@ gmail.com
Inverted papilloma involving the temporal bone is very rare and has been classified into two types-primary and secondary. ${ }^{7}$ Primary tumors of the middle ear have no history of associated sinonasal papillomas, whereas secondary tumors have a history of previous or concurrent sinonasal papillomas.

\section{CASE REPORT}

A 50-year-old female presented to our outpatient department with complaints of right-sided nasal obstruction for 6 months, which was insidious in onset, gradually progressive, not relieved on medication, and with no diurnal variation. She had associated blood-stained discharge from right nasal cavity for 3 months, which was spontaneous, minimal quantity, stopped spontaneously without any medical intervention. There was no associated anosmia, headache, or decreased vision.

She also complained of decreased hearing in right ear for 4 months, which was insidious in onset and gradually progressive. She could hear when spoken in raised voice but did not have difficulty in understanding speech. There was blood-stained discharge from right ear for 2 months, which was spontaneous and minimal in quantity. No complaints of giddiness, facial weakness, or history of trauma were noted.

She underwent two nasal surgeries in the past. In 2011, she underwent excision of the nasal mass elsewhere, the biopsy of which was reported as inverted papilloma of nose. In 2012, she had a recurrence of right sinonasal inverted papilloma, for which she underwent right endoscopic medial maxillectomy. She was symptom-free for 2.5 years.

On examination of the right ear under the microscope, a reddish polypoidal mass was seen in the bony part of the external auditory canal, which was bleeding on touch and insensitive to pain obscuring the tympanic membrane completely. The site of origin of the mass could not be identified. On rigid nasal endoscopy, a reddish, polypoidal, friable mass was seen occupying the right nasal cavity, which was bleeding on touch, insensitive to pain, and site of origin could not be identified. Septal perforation in the cartilaginous part was seen through which the mass was seen to bulge into the left nasal cavity. Rest of the ear, nose, and throat examination was normal. 
Routine blood investigations were done. Biopsy was taken from the polypoid mass in the right nasal cavity and in the right external auditory canal, both of which were reported as inverted papilloma. Pure tone audiogram showed a mixed hearing loss in right ear with airbone gap of $25 \mathrm{~dB}$. There was bilateral high-frequency sensorineural hearing loss. Tympanometry showed bilateral type "C" curve. Imaging of the nose and paranasal sinus with the temporal bone was done, which included computed tomography (CT) with contrast and magnetic resonance imaging (MRI) with contrast. The CT showed a homogeneous soft tissue density in the right nasal cavity, ethmoid sinus, maxillary sinus, eustachian tubal area, right middle ear, and mastoid. However, on T2-weighted MRI, there was a contrast-enhancing, hyperintense lesion limited to right nasal cavity and ethmoids, and also in the right middle ear suggestive of tumor, eustachian tubal area showed no contrast enhancement or hyperintense lesion, indicating it to be only edema (Figs 1 and 2).

Revision endoscopic clearance of the right sinonasal cavity was done. A posterior septotomy was done for better surgical access. Lobulated, pink, polypoidal mass was seen occupying the right nasal cavity and anterior and posterior ethmoids. The mass was attached to the right pterygoid process region posteriorly, for which the pterygoid process was drilled out for complete clearance of the lesion. Maxillary sinus and sphenoid sinus was free of the mass. After complete clearance from right sinonasal cavity, 70 nasal endoscope was used to visualize the right eustachian tube, to ensure that the tube was clear and there was no communication via the eustachian tube. Both the nasal cavities were packed with $8 \mathrm{~cm}$ Merocel pack. In the right ear, the mass was excised from the external auditory canal and a subtotal perforation of the
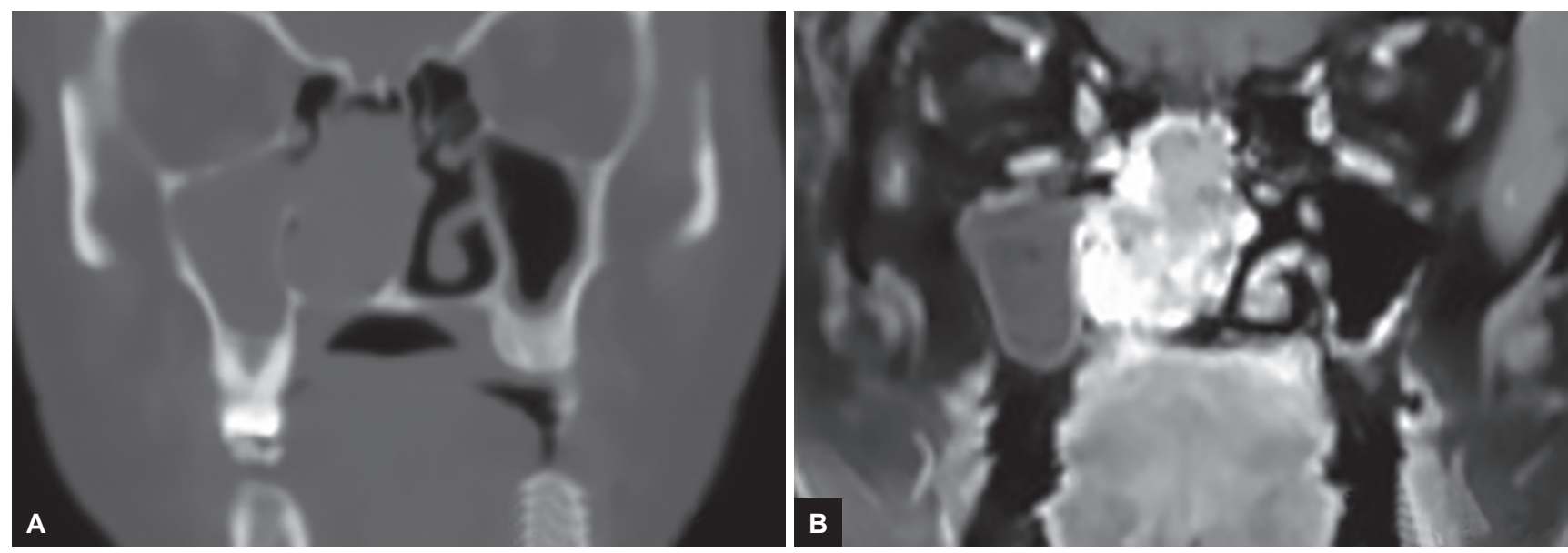

Figs 1A and B: (A) The CT paranasal sinus showing homogeneous soft tissue density involving the right nasal cavity, anterior ethmoids, and the right maxillary sinus; (B) MRI T2-weighted image of paranasal sinus showing a contrast-enhancing, hyperintense lesion limited to right nasal cavity and ethmoids. Right maxillary sinus is free of the mass
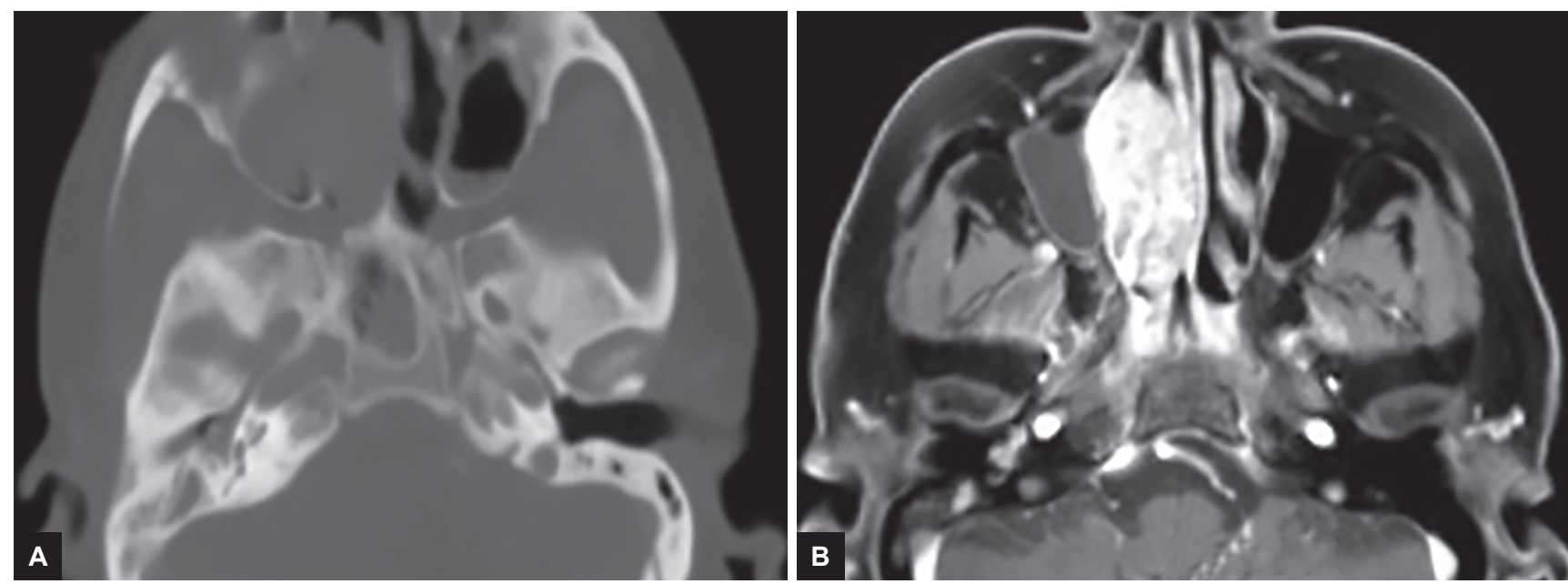

Figs 2A and B: (A) The CT paranasal sinus axial cuts showing homogeneous soft tissue density involving the right maxillary sinus, right nasal cavity extending up to nasopharynx, right eustachian tube, and also involving the right middle ear; (B) MRI T2-weighted image of same cut showing a contrast-enhancing, hyperintense lesion limited to right nasal cavity, nasopharynx, and pterygoid region and a separate contrast-enhancing lesion in the right middle ear. Eustachian tube is free of the lesion 


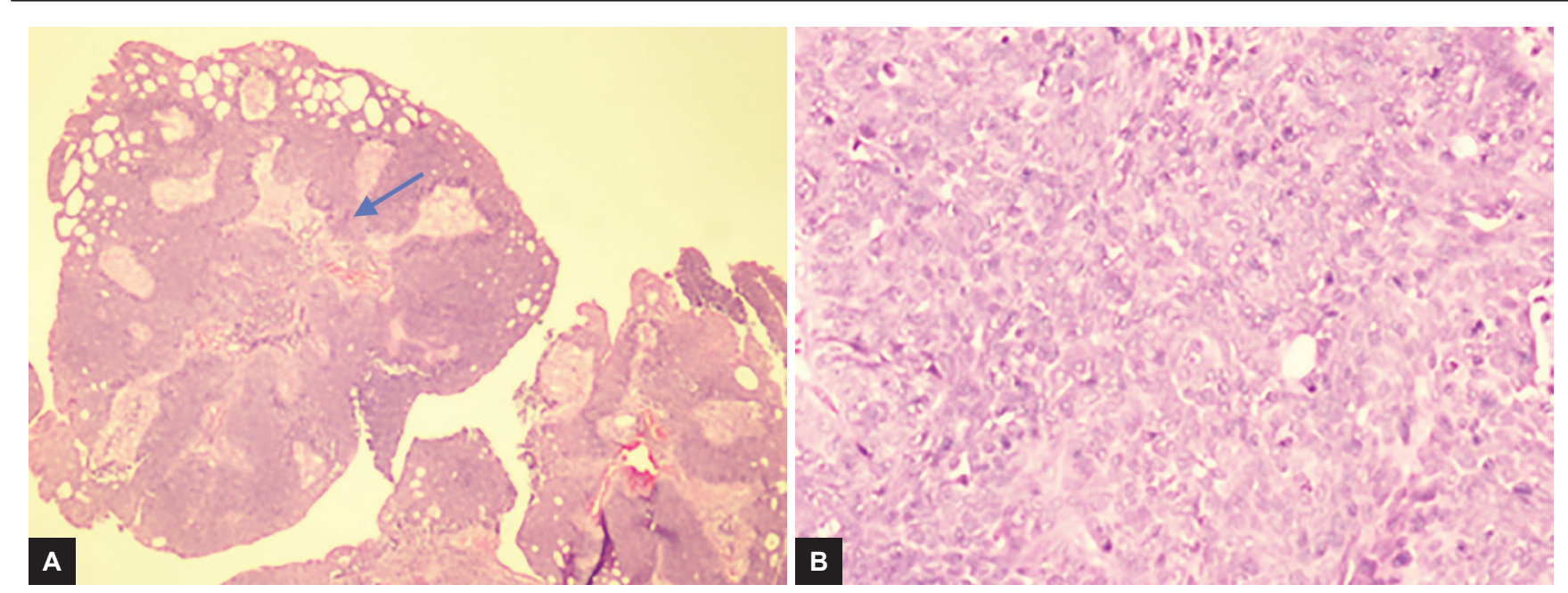

Figs 3 A and B: Low magnification (4x) hyperplastic ribbons of basement membrane-enclosed squamous and respiratory epithelium that grow endophytically into the underlying stroma with the absence of mucoserous glands; (B) high magnification (20x) inflammatory cells, especially neutrophils, transmigrate through the epithelium

tympanic membrane was visualized. Pink, friable, lobulated, polypoidal mass was seen completely occupying the middle ear, ossicles were not visible. A canal wall up mastoidectomy was done and as the mass was being debulked, ossicles were seen to be intact. But the malleus and incus had to be removed for complete clearance of the disease. The mass was seen to be arising from the anterior wall of middle ear, close the eustachian tubal orifice, but the tube was free of the lesion. Complete clearance of the lesion from the right middle ear and mastoid was done. Type III tympanoplasty was done.

Biopsy of the surgical specimen from the right nasal cavity, nasopharynx, right middle ear was sent for histopathological examination. Low magnification $(4 \times)$ showed hyperplastic ribbons of basement membrane enclosing squamous and respiratory epithelium that grew endophytically into the underlying stroma with the absence of mucoserous glands. On high magnification $(20 \times)$, the findings were confirmed. There were no features of malignancy (Fig. 3).

Postoperative period was uneventful and the patient was discharged. She is on regular follow-up for 1 year now and there is no recurrence till date.

\section{DISCUSSION}

Sinonasal inverted papilloma is more commonly seen in men (4:1 male-to-female ratio), whereas the review of the literature on inverted papilloma of the middle ear shows a 3:1 female-to-male ratio. ${ }^{8}$

The etiology of inverted papilloma of middle ear is not very well understood, as the middle ear mucosa is not of Schneiderian type. In this case, there was no evidence of direct spread through the eustachian tube, which usually forms a logical path for the nasal mucosal cells to spread into the middle ear. ${ }^{9-11}$
Various theories have been proposed in the literature for the development of inverted papilloma of temporal bone, which includes (a) direct spread of tumor from the sinonasal cavity via the eustachian tube, ${ }^{10,12}$ (b) primary middle ear involvement which is secondary to metaplastic change ${ }^{10,13}$ in the middle ear mucosa due to chronic irritation like chronic otitis media ${ }^{9}$ stimulating the development of Schneiderian mucosa in middle ear; (c) embryonic migration of ectopic Schneiderian membrane into the mucosa of the middle ear;, ${ }^{9,14}$ (d) surgical manipulation during removal of sinonasal tumor might seed tumor cells into extranasal regions. ${ }^{15}$ In this case, seeding of tumor during previous sinonasal surgeries might be a possible cause for multicentricity.

Inverted papilloma of temporal bone most commonly presents with complaints of hearing loss, otalgia, and otorrhea. In a patient having both sinonasal and temporal bone inverted papilloma, like in our case, the sinonasal variant usually precedes the temporal bone tumor. ${ }^{16}$ The clinical presentation and imaging of temporal bone Schneiderian papilloma are not very specific and the diagnosis can only be reliably made after histopathology of the biopsy or the surgical specimen. Magnetic resonance imaging with contrast plays a very important role in differentiating the tumor from edema.

Recently, endoscopic approaches are more preferred for excision of sinonasal inverted papillomas. Waitz and Wigand $\mathrm{d}^{17}$ concluded that endoscopic removal of sinonasal inverted papillomas can be achieved even for large lesions involving the posterior ethmoidal sinus, the nasofrontal duct, or sphenoid sinus, with recurrence rate of $17 \%$.

Treatment of inverted papilloma of the temporal bone, like the sinonasal cavity, should be focused on complete removal of the tumor. ${ }^{18}$ Inverted papilloma of the middle ear has a tendency for malignant transformation and 
recurrence as high as 35.3 and $56.25 \%$ respectively. ${ }^{19}$ Owing to the high rate of recurrences and extensive spread of middle ear inverted papilloma, prognosis is poor even after an aggressive surgical treatment. Adjuvant radiotherapy can be given in cases of recurrence, malignant transformation, or in cases of unresectable tumors. $^{20}$

\section{CONCLUSION}

We report this rare presentation of inverted papilloma with its unique characteristics of multicentricity, tendency to recur, and local aggressiveness in spite of its benign nature. In our patient, we assume that the tumor was multicentric in origin, as the imaging and intraoperative findings were unable to detect any continuity of lesions or any irregularity of the eustachian tubes. Owing to the high recurrence rate, chance of malignant transformation, absence of reliable prognostic markers, a strict postoperative follow-up is mandatory and which includes regular otomicroscopy, nasal endoscopy, and imaging.

\section{REFERENCES}

1. Skolnik EM, Loewy A, Friedman JE. Inverted papilloma of the nasal cavity. Arch Otolaryngol 1966 Jul;84(1):61-67.

2. van der Putten L, Bloemena E, Merkus P, Hensen EF. Schneiderian papilloma of the temporal bone. BMJ Case Rep 2013 Dec 5;2013.

3. Kramer R, Som ML. True papilloma of the nasal cavity. Arch Otolaryngol Head Neck Surg 1935 Jul;22(1):22-43.

4. Ringertz N. Pathology of malignant tumors arising in the nasal and paranasal cavities and maxilla. Acta Otolaryngol 1938;(Stockh) (Suppl): 27:31-42.

5. Calcaterra TC, Thompson JW, Paglia DE. Inverting papillomas of the nose and paranasal sinuses. Laryngoscope 1980 Jan;90(1):53-60.

6. Myers EN, Schramm VL, Barnes EL. Management of inverted papilloma of the nose and paranasal sinuses. Laryngoscope 1981 Dec;91(12):2071-2084.
7. Shen J, Baik F, Mafee MF, Peterson M, Nguyen QT. Inverting papilloma of the temporal bone: case report and meta-analysis of risk factors. Otol Neurotol 2011 Sep;32(7):1124-1133.

8. de Filippis C, Marioni G, Tregnaghi A, Marino F, Gaio E, Staffieri A. Primary inverted papilloma of the middle ear and mastoid. Otol Neurotol 2002 Jul;23(4):555-559.

9. Wenig BM. Schneiderian-type mucosal papillomas of the middle ear and mastoid. Ann Otol Rhinol Laryngol 1996 Mar;105(3):226-233.

10. Stone DM, Berktold RE, Ranganathan C, Wiet RJ. Inverted papilloma of the middle ear and mastoid. Otolaryngol Head Neck Surg 1987 Oct;97(4):416-418.

11. Pou AM, Vrabec JT. Inverting papilloma of the temporal bone. Laryngoscope 2002 Jan;112(1):140-142.

12. Jones ME, Wackym PA, Said-Al-Naief N, Brandwein M, Shaari CM, Som PM, Zhang DY, King WA. Clinical and molecular pathology of aggressive Schneiderian papilloma involving the temporal bone. Head Neck 1998 Jan;20(1):83-88.

13. Roberts WH, Dinges DL, Hanly MG. Inverted papilloma of the middle ear. Ann Otol Rhinol Laryngol 1993 Nov;102(11):890-892.

14. Kaddour HS, Woodhead CJ. Transitional papilloma of the middle ear. J Laryngol Otol 1992 Jul;106(7):628-629.

15. Miller PJ, Jacobs J, Roland JT, Cooper J, Mizrachi HH. Intracranial inverting papilloma. Head Neck 1996 Oct;18(5): 450-453; discussion 454.

16. Zhou H, Chen Z, Li H, Xing G. Primary temporal inverted papilloma with premalignant change. J Laryngol Otol 2011 Feb;125(2):206-209.

17. Waitz G, Wigand ME. Results of endoscopic sinus surgery for the treatment of inverted papillomas. Laryngoscope 1992 Aug;102(8):917-922.

18. Carlson ML, Sweeney AD, Modest MC, Van Gompel JJ, Haynes DS, Neff BA. Inverting papilloma of the temporal bone: report of four new cases and systematic review of the literature. Laryngoscope 2015 Nov;125(11):2576-2583.

19. Rubin F, Badoual C, Moya-Plana A, Malinvaud D, Laccourreye O, Bonfils P. Inverted papilloma of the middle ear. Eur Ann Otorhinolaryngol Head Neck Dis 2012 Aug;129(4):207-210.

20. Barbosa JL, Pinheiro SD, Freitas MR de, Nunes AAA, Leite EB. Sinonasal inverted papilloma involving the middle ear and the mastoid. Braz J Otorhinolaryngol 2012 Dec;78(6):122. 